\title{
Contrast discrimination between stimuli of orthogonal orientations
}

\author{
BERNT C. SKOTTUN \\ University of California, Berkeley, California
}

\begin{abstract}
Previous investigations have shown that spatial frequency discrimination performance does not suffer when observers are made to compare the frequency of orthogonal gratings. It remains to be determined if contrast discrimination is similarly independent of the relative orientations of the two gratings to be compared. Using a 2AFC method of constant stimuli, contrast discrimination was studied under conditions in which observers compared the contrast of two orthogonal gratings. Contrast discrimination thresholds determined in this way were compared with thresholds obtained with parallel stimuli. Discrimination thresholds obtained under the two conditions were similar.
\end{abstract}

Two recent investigations (Bradley \& Skottun, 1984; Burbeck \& Regan, 1983) have addressed the question of whether visual discrimination is degraded when the stimuli differ along other dimensions than the one to be discriminated. Specifically, these investigations asked if differences in spatial frequency affect orientation discrimination and, conversely, if differences in orientation affect spatial frequency discrimination. Neither of these changes along secondary stimulus dimensions were found to impair discrimination performance. It remains to be seen if such independence of secondary stimulus dimensions also holds for other kinds of visual discrimination. The present study investigated contrast discrimination between stimuli of different orientations. Observers were required to compare the contrast of orthogonal gratings. Contrast discrimination thresholds obtained in this way were compared with thresholds obtained with parallel stimuli.

It is possible that, because of astigmatism, meridional amblyopia, or anisotropies in the stimulus, the perception of contrast varies with stimulus orientation. If so, it would be possible for gratings of identical physical contrast to be discriminable. Therefore, to assess discrimination performance, it is necessary to determine contrast discrimination thresholds separately from any potential shifts in perceived contrast. Also, it is desirable to use an objective method to determine the discrimination thresholds. How to achieve both goals simultaneously is not obvious. One might want to combine a two-alternative forced-choice (2AFC) procedure with a method of fixed stimuli and have subjects judge which of two stimuli was of higher contrast. By plotting the percentage of presentations in which the test stimulus is judged to be of higher

I am indebted to Arthur Bradley for creative input and to Lori and Cathy Kodama for assistance with the manuscript. The work was supported by the Norwegian Research Council for Science and Humanities (NAVF).

The author's mailing address is: School of Optometry, University of California, Berkeley, CA 94720. contrast as a function of actual test stimulus contrast, a psychometric function can be obtained. Under normal circumstances, the slope of this psychometric function would provide a measure of contrast discrimination: the steeper the function, the lower the discrimination threshold. If there were no systematic differences in perceived contrast between the reference and test stimuli, the curve would cross the $50 \%$ level at the contrast of the reference stimulus. However, if perception of contrast in some way depended on orientation, and if one were to repeat this test with reference and test stimuli of different orientations, the resulting psychometric function would be shifted to either higher or lower contrasts. The slope of the function would still provide a measure of discrimination. Using test and reference stimuli that were uniquely associated with two different orientations (e.g., reference always vertical and test always horizontal), the observer would be able to identify the reference and test stimuli from the orientation of the grating.

To circumvent this problem, one might attempt to perform the test with random association between orientation and reference and test stimuli. If the reference and test stimulus orientation alternated so that each was, for example, vertical on half the trials and horizontal on the other half, the resulting psychometric function could be thought of as the "average" of the function for vertical reference and horizontal test gratings and horizontal reference and vertical test stimuli. Shifts in perceived contrast with orientation will shift one of these component functions toward lower and the other toward higher contrasts. The "average" of these will be a function that is centered (i.e., crosses the $50 \%$ correct response level) at the contrast of the reference stimulus but has a slope that is shallower than either component function alone. The reduced steepness would then erroneously be interpreted as reflecting increased discrimination thresholds.

For this reason, the experiment must be performed with a unique association between stimulus orientation and reference and test stimuli. By introducing a second set 
of reference and test stimuli, it is possible to prevent grating orientation from providing cues to the observer. Also in this second set are the two stimuli uniquely tied to stimulus orientation, but with the inverse relation. For example, one set of stimulus pairs would have reference stimuli of vertical orientation and horizontal test stimuli, while the second set of pairs would have horizontal reference and vertical test stimuli. When the stimulus pairs from the two sets are randomly interleaved, the subject will be unable to identify reference and test stimuli on the basis of orientation.

A psychometric function can be constructed from the responses to each set of stimuli, and separate objective assessments of discrimination performance and shifts in perceived contrast can be made by noting both the steepness and the lateral position of the functions.

\section{METHOD}

The goal of the investigation was to assess the effect of differences in orientation on contrast discrimination relative to discrimination between parallel stimuli. It was therefore necessary to also determine discrimination performance with parallel stimuli. Therefore, two additional psychometric functions were determined: one with both stimuli vertical and the other with both stimuli horizontal. As a result, a total of four psychometric functions were determined: (1) test, vertical; reference, vertical; (2) test, horizontal; reference, horizontal; (3) test, vertical; reference, horizontal; and (4) test, horizontal; reference, vertical. Stimuli from all four functions were interleaved randomly into one sequence. This procedure is essentially the same as that used by Bradley and Skottun (1984) to study spatial frequency discrimination between orthogonal gratings.

\section{Apparatus}

The stimuli consisted of 4-cycles/degree sinusoidal luminance gratings. These were generated on a CRT screen (Techtronics 606, P31 phosphor, mean luminance $45 \mathrm{~cd} / \mathrm{m}^{2}$ ) by a microprocessor-controlled stimulus generator (Milkman et al., 1978). The CRT screen was masked down to a circular field of $4^{\circ}$ diameter and was viewed (binocularly) from $114 \mathrm{~cm}$. Within each stimulus pair, each presentation lasted $0.5 \mathrm{sec}$ and was separated by a 0.5 -sec period of blank screen. The reference stimulus remained fixed at $25 \%$ contrast, but the test stimulus could be any one of 11 different contrasts ranging (in $1-\mathrm{dB}$ steps) from $14 \%$ to $50 \%$. Contrast was defined as

$$
C=(L \max -\mathrm{L} \min ) /(\mathrm{L} \max +\mathrm{Lmin}) .
$$

The subject's task was to indicate, by pressing one of two buttons, which of the two presentations contained the stimulus with the highest contrast. The response initiated the presentation of the next stimulus pair. A computer recorded the responses and randomized the sequence of stimuli.

\section{Subjects}

Two observers, the author and a paid subject (T.O.), participated in the experiment.

\section{RESULTS}

The results for the two observers are shown in Figures 1 and 2. The percentage of trials in which the test stimulus was seen as having higher contrast than the refer-

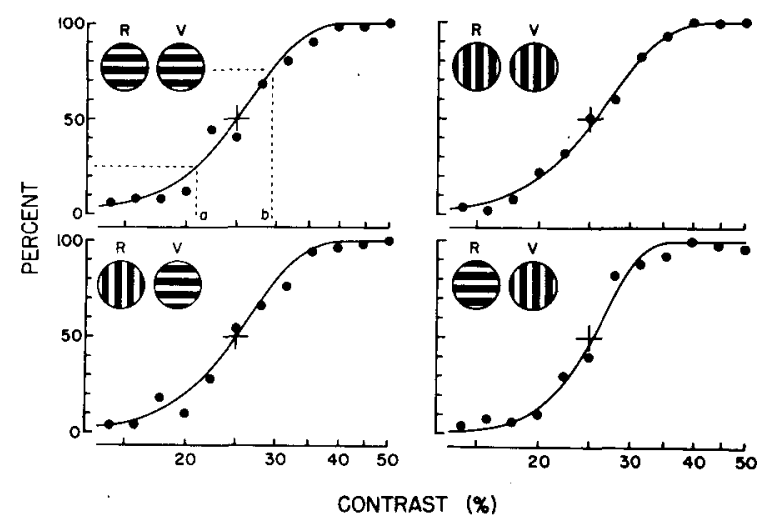

Figure 1. Psychometric functions for seeing the test stimulus as having higher contrast than the reference stimulus. Ordinates give the percentage of presentations for which test stimulus was seen as having higher contrast than the reference stimulus. The abscissae give the physical contrast of the test stimulus $[C=(L \max -L m i n) /$ (Lmax + Lmin)]. Each panel presents results for one combination of stimulus orientations. In each panel, the stimulus combination is symbolized in the insets ( $R=$ reference, $V=$ variable, $i . e .$, test). The cross in the center of each panel gives the contrast of the reference grating (25\%) and the $50 \%$ probability level. In the absence of any shifts in perceived contrast, curves will pass through the center of this cross. Smooth curves were fitted with a least squares fit procedure (see text). Dashed lines illustrate how the slopes of the functions were quantified (see text and Table 1). The figure gives results for Observer B.C.S. $(\mathbf{N}=\mathbf{5 0})$.

ence of $25 \%$ contrast is represented as a function of the actual contrast of the test stimulus. Each of the four panels gives the results for one combination of reference/test stimulus orientations (symbolized by the insets). Each data point represents the responses to 50 stimulus presentations. Smooth curves, $Y=1-\exp \left(-(x / a)^{b}\right)$ (Quick, 1974; Tolhurst et al., 1983), fitted to the data with a least squares fit procedure, show no systematic differences in the slopes for similar versus different orientations. This point is addressed quantitatively in Table 1 . The abscissae corresponding to the $75 \%$ and $25 \%$ levels (points $a$ and $b$ in Figure 1) were computed from the fitted curves, and the difference between these values were calculated. These

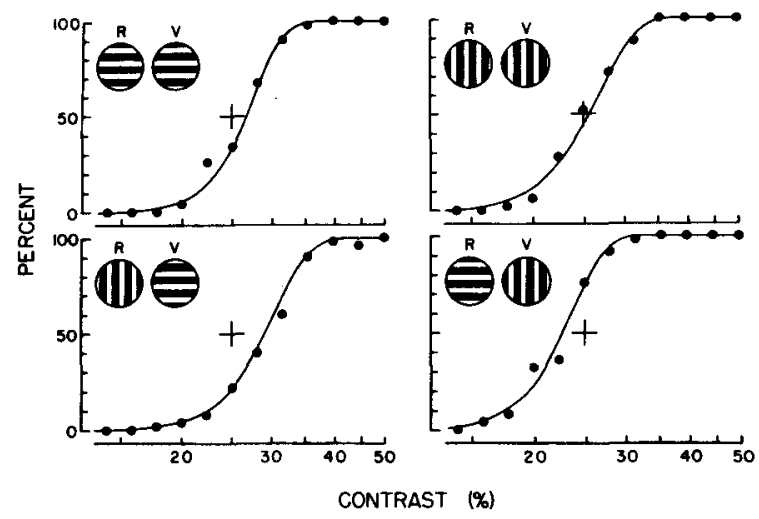

Figure 2. Same as Figure 1, but for Observer T.O. Note lateral shifts in the curves produced with orthogonal stimuli. 
Table 1

Quantifications of the Steepness of the Psychometric Functions

\begin{tabular}{lcccc}
\hline & \multicolumn{4}{c}{ Combinations of Orientations } \\
\cline { 2 - 5 } Observers & $\mathrm{HH}$ & $\mathrm{VV}$ & $\mathrm{HV}$ & $\mathrm{VH}$ \\
\hline B.C.S. & 3.01 & 3.05 & 2.16 & 2.94 \\
T.O. & 1.68 & 2.21 & 1.93 & 2.00 \\
\hline
\end{tabular}

Note-The abscissae corresponding to the psychometric functions' crossings of the $25 \%$ and $75 \%$ levels (i.e., $a$ and $b$ in Figure 1) were determined. The present values express the differences, in decibels $(I \mathrm{~dB}=$ $1 / 20$ log unit), between these two values. The smaller the difference, the steeper is the function. Each column gives results for one combination of stimulus orientations. The combination of stimuli for each column is indicated by the capital letters in the upper row $(H=$ horizontal and $V=v e r t i c a l)$, with the first letter denoting the reference and the second the test stimulus. The two rows give results for the two observers.

differences thus provide an inverse measure of steepnessthe steeper the curve, the smaller the difference. Table 1 gives the difference, in $\mathrm{dB}(1 \mathrm{~dB}=1 / 20 \log$ unit $)$, associated with each of the four stimulus combinations for each of the two observers. On average, for both observers, these values were 2.49 and $2.26 \mathrm{~dB}$ for similar and orthogonal orientations, respectively. The difference between these values is negligible, and would, if anything, indicate finer discrimination between orthogonal gratings.

As for shifts in perceived contrast with orientation, no systematic displacements of the functions are evident in the case of Observer B.C.S. (Figure 1). However, for Observer T.O. (Figure 2), some displacements can be seen. The data obtained with horizontal reference stimulus are shifted toward lower contrasts and, conversely, the data obtained with a vertical reference are displaced toward higher contrasts. It appears from these data that Observer T.O. perceived vertical gratings as having more contrast than horizontal ones. An alternative explanation is, of course, that the stimulus was anisotropic with regard to contrast. To test this possibility, the experiment was repeated with the oscilloscope rotated on its side. The shifts in the data did not change with rotation of the oscilloscope: data obtained with horizontal (relative to the eyes) reference stimuli remained shifted toward lower contrasts (the fitted curve crossed the $50 \%$ performance level at $23.4 \%$ contrast, as compared with $22.0 \%$ contrast in the unrotated condition) and data obtained with vertical reference stimuli remained shifted toward higher contrasts (the curve crossed the $50 \%$ level at $28.1 \%$ contrast, as compared with $28.6 \%$ contrast with the unrotated oscilloscope). Therefore, the shifts cannot be attributed to the stimulus, but must reflect an anisotropy in the visual system-perhaps some slight astigmatism. This interpretation was supported by the fact that T.O. also showed slightly higher contrast detection thresholds for horizontal gratings (about 1.07 times higher than those for vertical gratings). This interpretation conflicts with the clinical data: T.O. showed no astigmatism on clinical tests and was corrected to $20 / 15$ with hard contact lenses on both eyes. Because hard contact lenses would normally have removed any astigmatism, the shifts in the curves (and the elevated contrast thresholds for horizontal gratings) remain unexplained. These results serve to emphasize the need to separate shifts in perceived contrast from the magnitude of the discrimination thresholds.

For comparison with previous studies of contrast increment threshold, the increments needed to increase the performance from $50 \%$ to $75 \%$ were computed. These correspond to increment thresholds obtained with a $75 \%$ correct criterion. Increment thresholds, averaged for the two observers, were (expressed as Weber fractions) 0.136 and 0.126 for similar and orthogonal orientations, respectively. These values are compatible with previously reported data obtained with parallel stimuli (Campbell \& Kulikowksy, 1966; Legge, 1981).

\section{DISCUSSION}

These results demonstrate that contrast discrimination is as accurate when the observer compares the contrast of orthogonal gratings as when the comparison is between parallel stimuli. In being resistant to changes along a secondary stimulus dimension, contrast discrimination performance is similar to discrimination of orientations and spatial frequencies (Bradley \& Skottun, 1984; Burbeck $\&$ Regan, 1983). This indicates that the ability to extract information about one stimulus dimension without interference from differences along other dimensions may represent a general feature of information processing in the visual system.

Neurons in the primary visual cortex (V1) respond only to stimuli over select ranges of orientations (Hubel \& Wiesel, 1962, 1968). Therefore, two orthogonal stimuli will activate largely nonoverlapping populations of cortical neurons. The present findings therefore suggest that the visual system is able to compare, with high accuracy, information about contrast conveyed by different sets of cortical neurons. A recent study (Livingstone \& Hubel, 1984 ) indicated that some nonoriented cells in the primary visual cortex of primates have efferents that leave VI. It is thus possible that contrast information is conveyed by these cells. However, it is currently held that these neurons primarily subserve color vision (Livingstone \& Hubel, 1984).

\section{REFERENCES}

Bradley, A., \& Skottun, B. C. (1984). The effects of large orientation and spatial frequency differences on spatial discriminations. Vision Research, 24, 1889-1896.

BURBECK, C. A., \& REGAN, D. (1983). Independence of orientation and size in spatial discriminations. Journal of the Optical Society of America, 73, 1691-1694.

Camprell, F. W., Kulikowski, J. J. (1966). Orientational selectivity of the human visual system. Journal of Physiology (London), $187,437-445$.

HubEL, D. H., \& Wiesel, T. N. (1962). Receptive fields, binocular interaction and functional architecture in the cat's visual cortex. Journal of Physiology (London), 160, 106-154.

Hubel, D. H., \& Wiesel, T. N. (1968). Receptive fields and functional architecture of monkey striate cortex. Journal of Physiology (London), 195, 215-243. 
Livingstone, M. S., \& Hubel, D. H. (1984). Anatomy and physiology of a color system in the primate visual cortex. Journal of Neuroscience, 4, 309-356.

LEGGE, G. E. (1981). A power law for contrast discrimination. Vision Research, 21, 457-467.

Milkman, N., Shapley, R., \& Schick, G. (1978). Experimental applications. A microcomputer-based visual stimulator. Behavior $R e$ search Methods \& Instrumentation, 10, 539-545.
QuicK, R. F. (1974). A vector-magnitude model of contrast detection. Kybernetik, 16, 65-67.

Tolmurst, D. J., Movshon, J. A., \& Dean, A. F. (1983). The statistical reliability of signals in single neurons in cat and monkey visual cortex. Vision Research, 23, 775-785.

(Manuscript received February 22, 1985; revision accepted for publication August 9, 1985.) 